\title{
Searching for Optical Counterparts to High-Energy Neu- trino Sources with the Zwicky Transient Facility
}

\author{
Ludwig Rauch $^{1}$ \\ ${ }^{1}$ DESY, D-15735 Zeuthen, Germany
}

\begin{abstract}
The IceCube neutrino observatory has discovered a flux of extragalactic neutrinos. However, the origin of these neutrinos is still unknown. Among the possible candidates are Gamma-Ray Bursts (GRBs), Core-Collapse Supernovae (SNe), Active Galactic Nuclei (AGN) and Tidal Disruption Events (TDEs) - all are accompanied by a characteristic optical counterpart. The goal of this study is thus to identify the neutrino sources by detecting their optical counterparts with the Zwicky Transient Facility (ZTF). ZTF features a high cadence northern-sky survey enabling realtime correlation of optical transients with high-energy neutrino candidates. In this talk I will highlight the multimessenger potential of ZTF for an online neutrino correlation study with IceCube.
\end{abstract}

\section{Summary}

In the last decade, the IceCube Neutrino Observatory detected a diffuse flux of high-energy neutrinos [1]. Their origin, however, remains as yet unknown. Multi-messenger programs are one of the most promising methods to identify high-energy neutrino sources to resolve this question, as these programs are able to detect possible counterparts e.g. of electro-magnetic origin. In particular, potential astrophysical neutrino sources with a detectable optical counterpart include Gamma-Ray Bursts (GRBs) [2], Core-Collapse Supernovae (CCSN) [3], Active Galactic Nuclei (AGN) [4, 5] and Tidal Disruption Events (TDEs) [6]. These sources are transients or variables with potential observable optical counterparts on timescales of hours to months. Novel all-sky optical survey instruments are thus uniquely qualified to search for correlations with high-energy neutrino detections of IceCube.

The Zwicky Transient Facility (ZTF) [7] is a Samuel Oschin Schmidt telescope which features an exceptionally large 48 inch field-of-view. ZTF is able to scan the northern sky with a cadence of approximately 3 days in the $g$ and r-band. The large field-of-view enables high cadence observations across the entire northern sky which can be exploited not only for detections of fast-fading transients but covers also easily error contours for high-energy neutrino target-of-opportunity observation. While ZTF provides photometric light curves, the spectroscopic type of the transient is essential for discriminating between neutrino sources (e.g. CCSN) and background detections (e.g. SNIa). At the site of ZTF, located at the Palomar Observatory, a robotic spectograph, the Spectral Energy Distribution Machine (SEDM), for fast transient classification is used to follow up a large fraction of detected transients [8]. The joint effort of ZTF and SEDM aims for a complete catalogue of classified transients up to an apparent magnitude between 18 and 19 . 
To find optical transients in vicinity of the neutrino positions, the software package AMPEL [9] is used to select all ZTF alerts in spatial and temporal coincidence. AMPEL enables real-time monitoring and fitting of light curves, which will steadily increase the precision of the estimated explosion time and peak magnitude for a given transient. Combined with a requirement for a temporal overlap between the transient properties and neutrino detection, photometric redshift as well as the energy information of the neutrino, the decision to take a spectrum of a transient will be based on a maximum likelihood analysis to increase the probability to target viable neutrino sources.

The goal of combining the ZTF telescope and IceCube detector is twofold. First, neutrinos with the highest energies are published as a GCN notification and are followed-up in a target of opportunity program of ZTF. Due to the large field-of-view, the error circle of the order of $1 \mathrm{deg}^{2}$ is well contained within one pointing of ZTF. A second program is based on the online neutrino stream [10] which allows to target neutrinos with lower energies. These neutrinos suffer, however, from a larger atmospheric background contamination, requiring more sophisticated search strategies. AMPEL enables a real-time correlation analysis using a maximum likelihood method, which is able to consider the neutrino energy as well as the time and spatial correlation between the neutrino and the transient. Finally, we aim to collect a complete catalogue of all transients in the northern sky which is correlated to a neutrino detection up to the limiting magnitude of the SEDM with the aim to apply a neutrino-source stacking analysis to probe neutrino emission from entire source populations.

\section{References}

[1] Aartsen, M. G. et al. Evidence for High-Energy Extraterrestrial Neutrinos at the IceCube Detector. Science 342, 1242856 (2013). 1311.5238.

[2] Waxman, E. Cosmological gamma-ray bursts and the highest energy cosmic rays. Phys. Rev. Lett. 75, 386-389 (1995). astro-ph/9505082.

[3] Janka, H.-T., Langanke, K., Marek, A., Martinez-Pinedo, G. \& Mueller, B. Theory of Core-Collapse Supernovae. Phys. Rept. 442, 38-74, DOI: 10.1016/j.physrep.2007.02. 002 (2007). astro-ph/0612072.

[4] Atoyan, A. \& Dermer, C. D. High-energy neutrinos from photomeson processes in blazars. Phys. Rev. Lett. 87, 221102, DOI: 10.1103/PhysRevLett.87.221102 (2001). astro-ph/0108053.

[5] Atoyan, A. M. \& Dermer, C. D. Neutral beams from blazar jets. Astrophys. J. 586, 79-96, DOI: 10.1086/346261 (2003). astro-ph/0209231.

[6] Dai, L. \& Fang, K. Can tidal disruption events produce the IceCube neutrinos? Mon. Not. Roy. Astron. Soc. 469, 1354-1359 (2017). 1612.00011.

[7] Bellm, E., Kulkarni, S., Graham, M. \& et al. . Sub. to PASP .

[8] Blagorodnova, N. et al. The SED Machine: a robotic spectrograph for fast transient classification. 1710.02917.

[9] Nordin, J. et al. Ampel. https://github.com/AmpelProject (2019).

[10] Aartsen, M. G. et al. The IceCube Realtime Alert System. Astropart. Phys. 92, 30-41 (2017). 1612.06028. 